\title{
Resektion und Lebertransplantation bei hepatozellulärem Karzinom
}

\author{
Stefan A. Farkas Hans Jürgen Schlitt \\ Klinik und Poliklinik für Chirurgie, Universtätsklinik Regensburg, Deutschland
}

\author{
Schlüsselwörter \\ Hepatozelluläres Karzinom · Leber · Leberresektion · \\ Zirrhose $\cdot$ Lebertransplantation
}

\section{Zusammenfassung}

Hintergrund: Kurative Therapieverfahren für das hepatozelluläre Karzinom (HCC) sind die Resektion und die Lebertransplantation. Grundlage für die Therapieentscheidung ist bisher die Klassifikation der Barcelona Clinic Liver Cancer Group (BCLC). Methode: Basis dieser Arbeit ist eine selektive Literaturübersicht zur Therapie des HCC. Leitlinien verschiedener Fachgesellschaften wurden einbezogen und aktuelle Arbeiten zu neuen chirurgischen Techniken besonders berücksichtigt. Ergebnisse: Primär resektable HCCs sollen reseziert werden. Für die Resektion kommen alle Patienten in adäquatem Allgemeinzustand ohne Zirrhose oder mit ChildA-Zirrhose ohne ausgeprägte portale Hypertension in Betracht. Hierbei ist von entscheidender Bedeutung, dass über die Möglichkeit der Resektabilität in einem ausgewiesenen Leberzentrum befunden wird. Auf die Leber beschränkte Rezidive können erneut operiert oder für eine Lebertransplantation gelistet werden. Techniken zur erweiterten Leberresektion und laparoskopische Verfahren finden zunehmend Anwendung und müssen weiter evaluiert werden. Schlussfolgerung: Neben der Resektion ist die Lebertransplantation inzwischen eine Standardtherapie für HCCs in Zirrhose. Innerhalb der Mailand-Kriterien können nach der Transplantation 5-Jahres-Überlebensraten von 70-90\% erreicht werden. Allerdings erfordern der zunehmende Organmangel und die daraus resultierenden längeren Wartezeiten mit der Gefahr des Tumorprogresses ein Umdenken. So können Patienten, die für eine Transplantation infragekommen, nicht nur zum Bridging bis zur Transplantation reseziert werden, sondern auch, um eine biologische Stratifizierung durchzuführen. Unter engmaschigem Monitoring kann dann erst bei Auftreten eines Rezidivs eine "Rescue»-Transplantation durchgeführt werden. Jedoch werden auch Patienten mit fortgeschritteneren Tumoren, die nicht oder erst zu spät transplantiert werden können, zunehmend mit guten Ergebnissen reseziert. Somit könnten die Kriterien für eine Resektion im Rahmen der BCLC-Klassifikation erweitert werden.

\section{Keywords}

Hepatocellular carcinoma - Liver - Liver resection .

Cirrhosis · Liver transplantation

\section{Summary}

Resection and Liver Transplantation of Hepatocellular Carcinoma

Background: Curative strategies for hepatocellular carcinoma ( $\mathrm{HCC}$ ) are liver resection and transplantation. The Barcelona Clinic Liver Cancer Group (BCLC) classification is the basis for therapeutic stratification. Method: This overview is based on a selective literature search on therapeutic strategies for HCC. Guidelines of different societies were taken into account while new surgical approaches were especially evaluated. Results: Primary resectable HCCs should be resected. Most patients without cirrhosis qualify for resection. A liver resection should be considered for patients with Child A cirrhosis but without severe portal hypertension and a stable health status. It is of utmost importance that resectability is evaluated in a designated liver center. Intrahepatic tumor recurrences can also be re-resected or listed for liver transplantation. New techniques for extended liver resections or minimally invasive liver resections are commonly used and need to be studied further. Conclusion: In addition to liver resection, liver transplantation represents a standard therapy for HCC in cirrhosis today. Whilst observing selection criteria, 5-year survival rates of $70-90 \%$ can be achieved. However, increasing organ shortage leads to longer waiting times and thus to a higher risk of tumor progress. Thus, if possible, patients eligible for transplantation could be primarily resected as a bridging therapy as well as biological stratification. Liver transplantation should then be offered as a 'rescue transplantation' if recurrence under close monitoring occurs. However, even patients with tumors in an advanced stage for which transplantation is not possible or too late can be resected with encouraging results. Therefore, the resection criteria according to the BCLC classification could be extended.

\section{KARGER \\ Fax +497614520714 \\ Information@Karger.com}

www.karger.com (c) 2013 S. Karger GmbH, Freiburg

1662-6664/13/0292-0103\$38.00/0

Accessible online at:

www.karger.com/vim
PD Dr. Stefan A. Farkas, MBA

Klinik und Poliklinik für Chirurgie

Universitätsklinikum Regensburg

Franz-Josef-Strauß-Allee 11, 93053 Regensburg, Deutschland

stefan.farkas@ukr.de 


\section{Einführung}

Die Therapie des hepatozellulären Karzinoms (HCC) orientiert sich heute im Großen und Ganzen an der «Barcelona Clinic Liver Cancer Group (BCLC)»-Klassifikation, die das Ausmaß des Tumors, den Grad der Zirrhose (Child-Stadium) und auch den klinischen Zustand des Patienten berücksichtigt [1]. Einzig aus der BCLC-Klassifikation lassen sich direkt therapeutische Konsequenzen ableiten (Abb. 1). Prinzipiell stehen drei kurative Therapieverfahren zur Behandlung des HCC zur Verfügung: die chirurgische Resektion, die orthotope Lebertransplantation sowie die Tumorablation. In Abhängigkeit von der Größe, Lage und Anzahl der Tumoren, der Leberrestfunktion bzw. dem Allgemeinzustand und gegebenenfalls Komorbiditäten soll im Rahmen einer interdisziplinären Konferenz die individuelle Therapie festgelegt werden. Von entscheidender Bedeutung ist hierbei, dass die Möglichkeit der Resektabilität in einem Leberzentrum von einem erfahrenen Leberchirurgen evaluiert wird.

Für die Resektion kommen prinzipiell alle Patienten ohne Zirrhose und Patienten in adäquatem Allgemeinzustand mit Child-A-Zirrhose ohne wesentliche portale Hypertension in Betracht (BCLC-Stadium 0) [1, 2].

\section{Hepatozelluläres Karzinom ohne Zirrhose}

Primär resektable HCCs sollen reseziert werden [3-5]. Große Fortschritte und die Standardisierung in der Leberchirurgie in den letzten zwei Jahrzehnten haben dazu geführt, dass bei gesunder Leber Resektionsausmaße von bis zu $80 \%$ mit geringer Morbidität und Mortalität erreicht werden können.

Kriterien der Nichtresektabilität sind die nichtresektable extrahepatische Tumormanifestation, die allgemeine Inoperabilität aufgrund der Komorbidität des Patienten sowie die fehlende funktionelle Reserve [4]. Insbesondere die Größe des Tumors alleine ist nur in Ausnahmefällen ein Ausschlusskriterium; entscheidend ist die Lage des Tumors in Bezug zu den Gefäßen. Aber auch eine Infiltration von Lebervenen ist kein alleiniges Ausschlusskriterium, da diese rekonstruiert werden können. Besonders bei großen Tumoren können gut atypische Resektionen durchgeführt werden; hierbei wird möglichst viel Lebergewebe belassen und der Patient verliert nur wenig funktionelles Lebergewebe. In nichtzirrhotischen Lebern ist ein funktionelles Residualvolumen von $25 \%$ ausreichend; d.h., es können bis zu 75\% einer gesunden Leber reseziert werden. Hierbei ist zu berücksichtigen, dass das HCC selbst meist nicht mehr zur Funktion der Leber beiträgt und somit bei der Berechnung des notwendigen funktionellen Residualvolumens vernachlässigt werden kann. Deshalb ist von entscheidender Bedeutung, dass die Beurteilung der chirurgischen Resektabilität durch einen in der hepatobiliären Chirurgie erfahrenen Chirurgen im Rahmen einer interdisziplinären Konferenz erfolgt. Potenziell resektable Tumoren können bei Primärdiagnose aufgrund eingeschränkter funktioneller Reserve oder nicht möglicher R0-Resektion gegebenenfalls neoadjuvant behandelt werden (transarterielle Chemoembolisation, TACE). Hierfür eignet sich auch die operative Hypertrophieinduktion durch «in-situ splitting» mit Pfortaderligatur.

Bei nichtzirrhotischen Lebern beträgt die 5-Jahres-Überlebensrate allerdings nur 40-60\%, da hier meist eine aggressivere Tumorbiologie vorliegt. Prognostische Faktoren für ein Rezidiv in nichtzirrhotischen Lebern sind der Residualtumorstatus, die Gefäßinvasion und das Tumorgrading [6-8].

\section{Fibrolamelläres Karzinom}

Das fibrolamelläre Karzinom ist eine seltene Sonderform des HCC ohne zugrunde liegende Zirrhose oder Hepatitis mit besonderen pathologischen Merkmalen und aggressiver Tumorbiologie, die typischerweise bei jungen Patienten auftritt (Abb. 2, 3). Die wenigen publizierten Daten zeigen ein 5-Jahres-Überleben von $37-76 \%$. Allerdings liegt das rekurrenzfreie 5-Jahres-Überleben nur bei 18\%. Lymphknotenmetastasen sind ein negativer prognostischer Faktor. Das mediane
Abb. 1. Behandlungsstrategie von HCCs (modifiziert nach BCLC) (PS = Performancestatus).

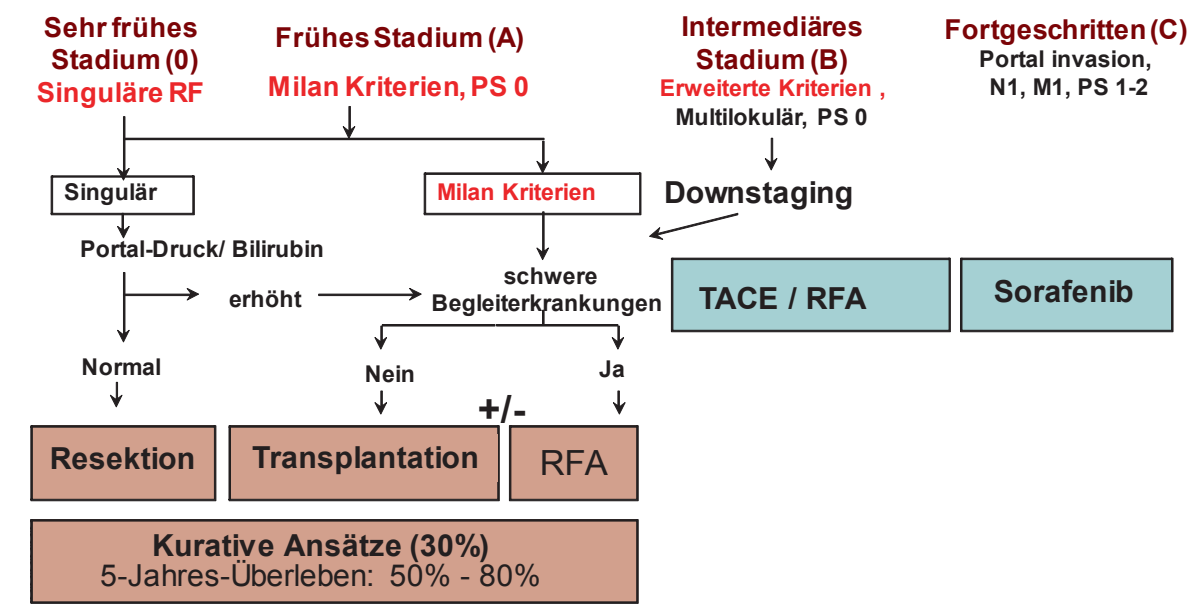

Farkas/Schlitt 

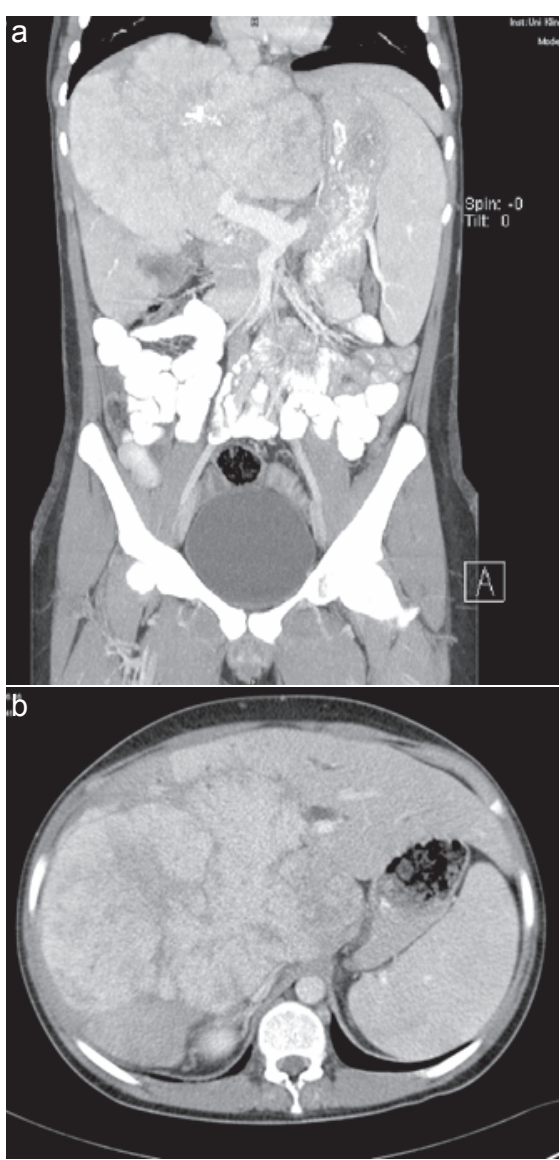

Abb. 2.a,b. Große fibrolamelläres Karzinom bei einem

18-jährigen Patienten.

Überleben ohne Resektion liegt bei 12 Monaten [9]. Da späte Rezidive häufig sind, sollten auch Re-Resektionen durchgeführt und gegebenenfalls - bei fehlendem Lymphknotenbefall - auch eine Transplantation erwogen werden, da keine anderen effektiven Therapiestrategien für das fibrolamelläre Karzinom bestehen [10]

\section{Leberresektion in Zirrhose}

Die Resektion eines HCC in Zirrhose setzt eine sorgfältige Patientenevaluation voraus. Für die Resektion des HCC kommen Patienten in gutem klinischem Allgemeinzustand mit Child-A-Zirrhose in Betracht (BCLC-Stadium 0) [3-5].

In Einzelfällen können auch Patienten mit Child-B-Zirrhose reseziert werden. Das Vorliegen einer portalen Hypertension (Aszites, Thrombozyten $<100$ 000, Splenomegalie) ist kein alleiniges Ausschlusskriterium für die Resektion, erhöht jedoch das operative Risiko. Hierbei muss im Hinblick auf das Ausmaß der portalen Hypertension im Einzelfall eine NutzenRisiko-Analyse der Resektion als alleiniger kurativer Therapieansatz durchgeführt werden. Im Zweifelsfall kann hierfür eine explorative Laparoskopie zur makroskopischen Beurteilung der Leber hilfreich sein. Insbesondere das präoperative Vorhandensein von Aszites weist jedoch auf einen protrahierten und somit komplizierteren postoperativen Verlauf hin.
Abb. 3.a. Resektionspräparat. b Lymphknotendissektion im Hilus.
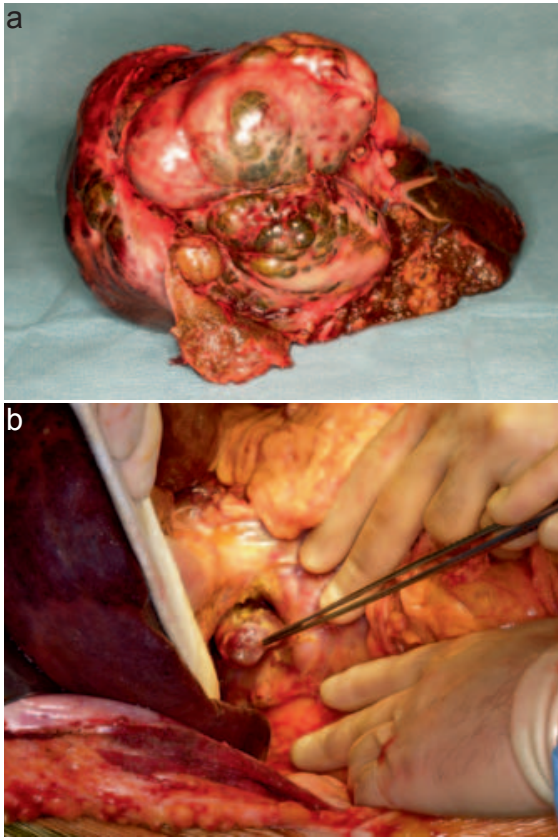

Die funktionelle Resektabilität soll die ausreichende postoperative Leberfunktion und die portale Hypertension berücksichtigen. Bei einer Child-A-Zirrhose werden mehr als $40 \%$ des Leberparenchyms benötigt. Verschiedene Verfahren zur Evaluation der Leberfunktion über das Child-Pugh-System hinaus - wie der Indocyanin-Grün-Test oder der Limax-Test - sind entwickelt worden, konnten sich aber bisher in der Routine noch nicht durchsetzen. Um möglichst parenchymsparend zu operieren, ist die Durchführung nichtanatomischer Resektionen sinnvoll, da sich kein Unterschied bezüglich der Rezidivhäufigkeit gezeigt hat. Letztendlich ist für die Resektabilität die Einschätzung eines erfahrenen Leberchirurgen in enger Absprache mit dem Hepatologen entscheidend.

\section{Notwendigkeit der optimierten Bildgebung zur Operationsplanung}

Zur Planung der operativen Therapie ist eine optimierte Bildgebung notwendig. Standard ist - über das CT-Thorax/ -Abdomen zum Staging hinaus - ein 3-Phasen-CT der Leber. Weiterhin ist als komplementäre Bildgebung eine dynamische Magnetresonanztomographie der Leber mit leberspezifischem Kontrastmittel sinnvoll. Der Ultraschall bzw. kontrastmittelverstärkte Ultraschall (contrast-enhanced ultrasound, CEUS) soll komplementär durchgeführt werden [11]. Darüber hinaus dient eine Fluor-18-Fluorodesoxyglukose-Positronenemissionstomografie (FDG-PET) sowohl zur Detektion extrahepatischer Metastasen als auch zur prognostischen Evaluation des HCC, da es den Differenzierungsgrad des HCC reflektiert $[12,13]$. Leider ist das PET bei eher gut differenzierten und kleinen HCCs negativ, sodass es als ScreeningMethode ungeeignet ist. 


\section{Intraoperativer Ultraschall ist Standard}

Ein intraoperativer Ultraschall (IOUS) ist unbedingt notwendig, um weitere Herde zu detektieren und um die Operationsstrategie optimal festlegen zu können. An unserem Zentrum haben wir insbesondere sehr gute Erfahrungen mit intraoperativem kontrastmittelgestütztem Ultraschall (intraoperativer CEUS) mit linearer Sonde gemacht. Hiermit konnten wir intraoperativ $50 \%$ mehr Herde als im alleinigen B-Bild finden [14, 15]. Diese Methode wird derzeit weiter evaluiert.

\section{Großer Sicherheitsabstand zum Tumor nicht unbedingt notwendig}

Bei einer potenziell kurativen Resektion des HCC hat der früher geforderte weite Sicherheitsabstand von $2 \mathrm{~cm}$ in Studien keinen Vorteil bezüglich eines lokalen Rezidivs gezeigt [16] Allerdings konnte in einer anderen Studie speziell bei kleineren Tumoren $(\mathrm{HCC}<2 \mathrm{~cm})$ gezeigt werden, dass der größere Sicherheitsabstand vor allem auch beim Vorliegen von Mikrometastasen einen Überlebensvorteil bringt [17]. Ursächlich für diese unterschiedlichen Ergebnisse ist möglicherweise eine unterschiedliche Tumorbiologie, da langsam wachsende große Tumoren oft ein weniger aggressives Wachstum haben als kleinere Tumoren mit Satellitenherden oder Mikrometastasen. Ein weiterer «Sicherheitsabstand» zur Resektionsfläche kann auch durch zusätzliche intraoperative Thermoablation erreicht werden. Eine zu erwartende R1-Situation hiernach ist somit keine Kontraindikation für eine Resektion. Daher sind speziell bei größeren Tumoren auch erweiterte Resektionen mit nur geringem Abstand oder gegebenenfalls auch mit einer R1-Situation hin zu einer zu erhaltenden Lebervene sinnvoll.

\section{Erneute Resektion von auf die Leber beschränkten Rezidiven}

Auch Re-Resektionen bei Rezidiven sind sinnvoll, da hier ebenfalls, insbesondere bei nicht extrahepatischem Tumorwachstum, 5-Jahres-Überlebensraten von bis zu $80 \%$ erreicht werden können. Diesbezüglich konnte in einer großen multivariaten Analyse gezeigt werden, dass die Re-Resektion die Therapie der Wahl bei Patienten ist, bei denen primär ein Herd reseziert wurde, die ein Jahr lang rezidivfrei waren und die keine portale Infiltration zeigen [18].

\section{Ergebnisse nach Resektion von solitären oder kleinen HCCs in Zirrhose}

Die besten Ergebnisse finden sich nach der Resektion von kleinen und solitären HCC-Herden in Zirrhose. In selektionierten Patientengruppen kann hiermit ein 5-Jahres-Überleben von $30-50 \%$ erreicht werden [19-21]. In einer aktuellen japanischen Registeranalyse [2] mit mehr als 20000 Patienten lag das rezidivfreie 10-Jahres-Überleben bei $22 \%$. Hierbei zeigten sich die Tumorgröße $(>5 \mathrm{~cm}$ ) und die Differenzierung als prognosebestimmend [2]. In anderen Studien gelten neben der Tumorgröße ein Alpha-Fetoprotein (AFP) > $1000 \mu \mathrm{g} / \mathrm{l}$, das Vorliegen einer R1-Situation nach Resektion sowie eine mikrovaskuläre Infiltration als Risikofaktoren für ein Tumorrezidiv [22, 23]. Da unter anderem die mikrovaskuläre Infiltration nicht präoperativ bestimmt werden kann, sind hier neue diagnostische Tools notwendig, um eine bessere Prognose- und somit Therapiebestimmung durchführen zu können. Das FDG-PET ist in diesem Zusammenhang ein möglicher Ansatz [13].

\section{Leberresektion versus Radiofrequenzablation in Zirrhose}

Entsprechend der aktuellen Datenlage kann keine eindeutige Aussage zugunsten der Resektion oder Radiofrequenzablation (RFA) getroffen werden. Beide Methoden sind zur Therapie kleiner HCCs $(<5 \mathrm{~cm})$ in zirrhotischen Lebern akzeptabel. Eine aktuelle Metaanalyse zeigt, dass die Resektion im Vergleich zur RFA mit signifikant besserem Gesamt- und rezidivfreiem Überleben assoziiert ist. Allerdings findet sich nach einer Resektion auch eine signifikant höhere Komplikationsrate. Bei HCC-Herden $>3 \mathrm{~cm}$ sollte der RFA eine Embolisation vorgeschaltet werden. Aufgrund des Risikos einer peritonealen Tumoraussaat bei subkapsulär gelegenen HCCs ist hier die Operation vorteilhaft. Zentrale kleine Läsionen in Zirrhose sind hingegen teilweise einfacher mittels RFA zu therapieren.

\section{Neue chirurgische Techniken für erweiterte oder weniger invasive Leberresektionen}

\section{Erweitertes Leberresektionsverfahren «in-situ split»: Pfortaderligatur und Parenchymdurchtrennung zur schnellen Hypertrophieinduktion}

Bis dato standen verschiedene Methoden zur Verfügung, um aus nichtresektablen resektable Tumoren zu machen. Zum einen gibt es die Möglichkeit der zweizeitigen Resektion bei multifokalen Tumoren. Hierbei wird zunächst der Tumor in einem Leberlappen reseziert und anschließend nach entsprechender Regeneration die zweite Seite operativ angegangen. Eine andere Möglichkeit ist die radiologische Pfortaderembolisation oder die operative Pfortaderligatur der tumortragenden Leberseite, sodass die kontralaterale Seite an Volumen und funktionalem Gewebe zunehmen kann. Auf diese Weise erreicht man eine Hypertrophie von etwa $10-46 \%$ in einem Zeitraum von 2-8 Wochen und eine Resektabilität von 70-100\% in ausgewählten Fällen [24, 25]. Problematisch ist allerdings, dass bis $\mathrm{zu} 40 \%$ der Patienten aufgrund des langen Zeitraums zwischen Pfortaderembolisation und Resektion einen Tumorprogress zeigen, der einen kurativen Ansatz bzw. eine Resektion verhindert. 
Am Universitätsklinikum Regensburg wurde, gemeinsam mit anderen Universitätskliniken, nun ein neues Verfahren evaluiert, bei dem die Pfortaderligatur mit einer kompletten Parenchymdissektion entlang des Ligamentum falciforme hepatis kombiniert wird (sogenanntes «in-situ split»). Hierbei wurde bei den 25 ausgewerteten Patienten vor der Komplettierungsoperation nach 9 Tagen eine mediane Volumenzunahme des linkslateralen Segments von 310 auf $536 \mathrm{ml}$ gemessen. Dies entspricht im Median einem Anstieg um 74\%. Die Überlebensrate im Gesamtkollektiv betrug nach einjähriger Follow-up-Periode 83\% [26]. Diese Technik wurde nun durch andere Zentren bestätigt und findet mittlerweile in einigen Leberzentren Anwendung [27, 28], dies auch in Kombination mit laparoskopischer Resektionstechnik [29]. Die langfristigen Ergebnisse müssen weiter evaluiert werden.

\section{Laparoskopische Leberresektion}

Laparoskopische Leberresektionen finden zunehmend bei ausgewählten Patienten Anwendung. Hierbei werden rein laparoskopische, laparoskopisch assistierte und handassistierte Techniken voneinander unterschieden. Mit allen drei Methoden können in erfahrenen Leberzentren mit entsprechender laparoskopischer Expertise gute onkologische Langzeitergebnisse erreicht werden. Allerdings existiert eine deutliche Lernkurve für größere laparoskopische Leberresektionen.

Beim laparoskopischen Vorgehen besteht nicht nur ein kosmetischer Vorteil, sondern vor allem Patienten mit Zirrhose können von dem kleineren Zugangsweg profitieren. Dies gilt insbesondere für die postoperative Aszitesbildung mit konsekutiver Fasziendehiszenz, die bei Child-A-Zirrhose auch ohne portale Hypertension häufiger auftritt. Darüber hinaus können auch notwendige Rezidiveingriffe aufgrund der geringeren Verwachsungen möglicherweise leichter durchgeführt werden. Dies trifft auch für Patienten zu, die nach Resektion aufgrund eines Rezidivs innerhalb der Mailand-Kriterien transplantiert werden müssen. In einer aktuellen Studie fand sich bei Patienten, die nach initial laparoskopischer Leberresektion wegen eines Rezidivs transplantiert werden mussten, eine geringere Operationszeit und ein geringerer Blutverlust.

In einer aktuellen Metaanalyse wurden 29 Fallserien mit insgesamt mehr als 2600 Patienten untersucht [30]. Hierbei handelte es sich um selektionierte Patienten zumeist ohne Zirrhose, bei denen sich ähnliche Ergebnisse wie nach konventioneller Resektion zeigten. Die spezifische Erfahrung des Chirurgen beeinflusste die Ergebnisse deutlich, sodass eine Lernphase als notwendig angesehen wird. Die handassistierte und laparoskopisch assistierte Technik war mit einem besseren perioperativen Outcome assoziiert. Von den drei oben beschriebenen Techniken sollte somit die rein laparoskopische Technik dem erfahrenen Chirurgen vorbehalten sein. Die laparoskopisch assistierte Technik wurde vor allem in zirrhotischen Lebern, bei Tumoren mit ungünstiger Lokalisation und bei der Leber-Lebendspende verwendet.
Daten zum langfristigen onkologischen Outcome liegen nur in wenigen Studien vor. Hierzu hat die Gruppe um Cherqui Ergebnisse von drei europäischen Zentren zu Patienten mit HCC zusammengefasst [31]. In einer prospektiven Datenanalyse wurden 163 laparoskopische Leberresektionen untersucht. Von diesen Patienten hatten 120 (73\%) eine Zirrhose. Bei 95\% der Patienten wurde ein rein laparoskopisches Vorgehen gewählt. Die mediane Tumorgröße lag bei $3,7 \mathrm{~cm}$. Es zeigte sich auch hier, dass nach einer gewissen Lernkurve die Komplikationen geringer und das Langzeitüberleben besser waren. In der aktuelleren Patientengruppe betrug das Gesamtüberleben nach 1, 3 und 5 Jahren jeweils 92,6, 68,7 und 64,9\%, und das rezidivfreie Überleben belief sich auf 77,5, 47,1 bzw. 32,2\%.

In einer aktuellen Zentrumsanalyse wurden das offene und das laparoskopische Vorgehen zumeist bei Patienten mit Zirrhose verglichen. Hier zeigten sich - allerdings bei kleiner Fallzahl - ein geringerer Blutverlust, weniger Komplikationen und ein kürzerer Krankenhausaufenthalt bei gleichem Langzeitüberleben [32].

Allerdings gibt es Grenzen für laparoskopische Resektionen. Dies sind vor allem Tumoren in den kranial gelegenen Lebersegmenten IVa, VII und VIII. Darüber hinaus sind große, sehr hilusnah gelegene oder an die Vena cava heranreichende Tumoren für das laproskopische Vorgehen weniger geeignet.

Weitere Studien werden die Komplikationen, das Langzeitüberleben und die ökonomischen Faktoren (längere Operationszeiten und teureres Equipment) in größeren Patientenkollektiven erfassen müssen.

\section{Lebertransplantation für hepatozelluläres Karzinom in Zirrhose}

\section{Gesichertes HCC in Zirrhose innerhalb der «Mailand- Kriterien» als Indikation für Lebertransplantation}

Basierend auf internationalen Studiendaten ist die Lebertransplantation bei der Anwendung von restriktiven Kriterien mit 5-Jahres-Überlebensraten von 70\% assoziiert [33-35]. Darüber hinaus wird auch die zugrunde liegende Zirrhose behoben, die ebenfalls für die Entstehung des Tumors verantwortlich ist und zudem ein eigenes Mortalitätsrisiko mit sich bringt. Für die Transplantation geeignet sind unter anderem Patienten, die primär aufgrund eines Child-Stadiums B-C oder einer ausgeprägten portalen Hypertension nicht reseziert werden können (BCLC-Stadium A-C). Der Mangel an postmortalen Organspendern macht jedoch eine Selektion der Patienten hinsichtlich ihres Überlebens notwendig. Bei Vorliegen von extrahepatischer Tumormanifestation oder makroskopischer Gefäßinvasion ist eine Lebertransplantation kontraindiziert [3-5].

Die besten Prädiktoren für das Überleben von Patienten mit HCC nach Lebertransplantation sind die Mailand-Kriterien [33-37]. Patienten, die die Mailand-Kriterien (ein Knoten $<5 \mathrm{~cm}$ oder bis zu 3 Knoten $<3 \mathrm{~cm}$, Abwesenheit von makro- 
Abb. 4. Wochen nach erweiterter Leberresektion mit Gefäßrekonstruktion.

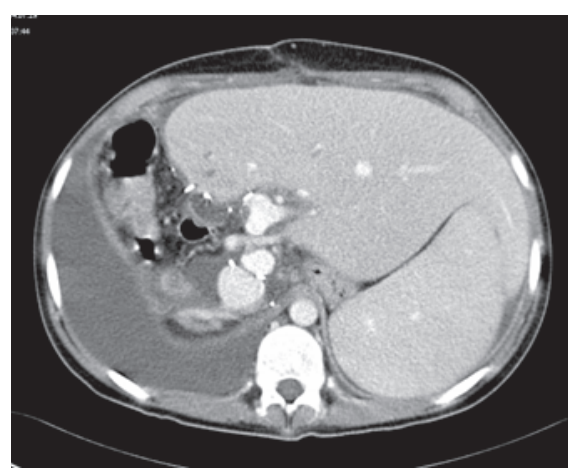

vaskulärer Infiltration) (Abb. 4) erfüllen und keine extrahepatischen Metastasen aufweisen, können im EurotransplantBereich für eine Lebertransplantation gelistet werden. Sie erhalten hierfür eine standardisierte, akzeptierte Sonderregelung. Hierbei muss der Tumor durch mindestens eine der drei folgenden Methoden nachgewiesen werden:

- Biopsie;

- AFP > 400 und Hypervaskularisation in einer Bildgebung;

- Hypervaskularisation in zwei Bildgebungsverfahren.

Diese so selektionierten Patienten erreichen 5-JahresÜberlebensraten von bis zu 70\% und eine Lokalrezidivrate von unter $15 \%$. Patienten außerhalb der Mailand-Kriterien können zwar gelistet werden, erhalten die Sonderregelung für HCC jedoch nicht. Weitere prognostische Kriterien sind die sogenannten UCSF(University of California San Francisco)Kriterien. Diese fordern eine Tumorgröße $<6,5 \mathrm{~cm}$ bei einem solitären Herd, weniger als 3 HCC-Herde mit einem Maximaldurchmesser des größten Herdes von $<4,5 \mathrm{~cm}$ oder eine maximale Summe der addierten Tumordurchmesser $<8 \mathrm{~cm}$. Auch mit diesen Kriterien kann eine 5-Jahres-Überlebensrate von bis zu 70\% erreicht werden [38]. Damit ist die Überlebenswahrscheinlichkeit der Patienten nach Transplantation ungefähr mit derjenigen von Patienten vergleichbar, die an einer Leberzirrhose ohne HCC leiden.

\section{Sonderregelung für Patienten mit HCC innerhalb der Mailand-Kriterien}

Die Kriterien für HCC-Patienten zur Transplantation sind aktuell im deutschen Transplantationsgesetz und bei der Allokation durch Eurotransplant verbindlich verankert. HCCPatienten innerhalb der Mailand-Kriterien erhalten dabei eine standardisierte, akzeptierte Sonderregelung, die zu einem sukzessiven Punktgewinn und somit de facto einem Vorrücken auf der Warteliste auch bei stabiler Organfunktion führt. Ansonsten werden Lebern im Eurotransplant-Bereich nach dem MELD-System (Model for End-Stage Liver Disease; kalkulierter Wert aus Kreatinin, Bilirubin und International Normalized Ratio) alloziert. Somit konnte ein HCCPatient mit stabiler Organfunktion mit einer Wartezeit von nur ungefähr einem Jahr rechnen. Allerdings hat sich aufgrund der dramatischen Verschlechterung der Organspendesituation in den letzten Jahren besonders in Deutschland die Wartezeit für diese Patienten deutlich verlängert. Außerdem wurden aufgrund der Möglichkeit der Sonderregelung auch mehr Patienten mit HCC für die Lebertransplantation gelistet, was wiederum zu einer Zunahme des Organmangels führt.

\section{Downstaging, Tumorkontrolle und «Bridging» bis zur Lebertransplantation durch Resektion, TACE und RFA}

Bei Patienten mit nichtresektablem HCC und bestehender Indikation zur Transplantation, aber fehlendem adäquatem Spenderorgan stellt die Wartezeit vor der Lebertransplantation ein hohes Risiko dar, aufgrund von Tumorprogression aus den engen Transplantationskriterien, die für Tumorgröße und -anzahl gelten, herauszufallen (Drop-out) [39]. Für das HCC innerhalb der Mailand-Kriterien korreliert daher die Drop-outRate mit der Länge der Wartezeit und der Größe des Tumors. Bei Patienten mit 2-3 HCC-Herden oder einem singulären HCC $>3 \mathrm{~cm}$ berichten Yao et al. [38] über Drop-out-Raten nach 6, 12 und 18 Monaten von 12, 56 und $89 \%$ gegenüber solchen von 0,10 und $21 \%$ bei einem singulären $\mathrm{HCC}<3 \mathrm{~cm}$. Zum Überbrücken (Bridging) der Wartezeit - und mit dem Ziel, den Tumor innerhalb der Transplantationskriterien zu halten - haben sich in den letzten Jahren ablative Verfahren (RFA) sowie die TACE etabliert. Sie haben den Vorteil einer geringen Invasivität, gelten als sicher sowie parenchymschonend und sind mehrfach wiederholbar. Patienten mit HCC auf der Warteliste müssen alle 3 Monate mit einem schnittbildgebenden Verfahren bezüglich des Tumorprogresses kontrolliert werden. Hierbei wird zum einen die Wirksamkeit der BridgingVerfahren und zum anderen ein Progress des Tumors untersucht. Wenn der nicht kontrollierbare Tumorprogress einen Drop-out aus den Mailand-Kriterien bedingt, muss der Patient von der Warteliste genommen werden. Somit werden Patienten mit einer aggressiveren Tumorbiologie, die nach einer Transplantation auch ein deutlich schlechteres Überleben als die anderen Patienten hätten, tumorbiologisch selektioniert.

\section{Leber-Lebendspende als Option}

Durch eine Leber-Lebendspende lässt sich die Wartezeit vermeiden und damit der mögliche Tumorprogress verhindern. Darüber hinaus entlastet dies den limitierten Pool an postmortalen Spenderorganen. Da das potenzielle Komplikationsrisiko für den Spender in erfahrenen Zentren relativ gering ist, sollte diese Möglichkeit bei Vorhandensein eines geeigneten Spenders evaluiert werden. Die Komplikationen und das Überleben nach Lebendspende sind für den Empfänger mit der postmortalen Spende vergleichbar. Allerdings kann die Lebendspende - im Gegensatz zur postmortalen Spende 
- beim klinisch stabilen Patienten zum optimalen Zeitpunkt erfolgen und bietet daher einen entscheidenden Vorteil gegenüber der postmortalen Spende (Abb. 4).

\section{Rezidivrisiko und Nachsorge}

Nach einer Lebertransplantation wird durch die notwendige lebenslange Immunsuppression mit Calcineurininhibitoren das Rezidivrisiko erhöht. Die Ergebnisse der multinationalen Silver-Studie mit mTOR-Inhibitor als antitumoröser Immunsuppression sind abzuwarten [40, 41].

Patienten mit HCC und Lebertransplantation müssen engmaschig (im ersten Jahr alle 3 Monate, dann jährlich) mittels Bildgebung und AFP nachgesorgt werden.

\section{Primäre Transplantation versus primäre Leberresektion und Transplantation als «Rescue»- Therapie}

Wie bereits beschrieben, ist die Lebertransplantation theoretisch die beste Therapie für HCCs innerhalb der MailandKriterien. Allerdings ist die Verfügbarkeit von postmortalen Organen limitiert und das Risiko eines Tumorprogresses aufgrund der längeren Wartezeiten nicht unerheblich. Besonders in Deutschland ist der Organmangel eklatant.

Eine mögliche Resektion des Tumors bei Patienten mit Child-A-Zirrhose als initiale Therapie vor der Listung zur Lebertransplantation hat folgende Vorteile: Zum einen kann diese als primäre Therapieoption unabhängig von der Zirrhose fungieren; zum anderen können durch die vollständige histologische Aufarbeitung eine Gefäßinvasion und Mikrosatellitenherde detektiert werden. Dies dient wiederum der Selektion der Patienten auf der Warteliste. Darüber hinaus ist die Resektion sofort verfügbar und bietet eine optimale Tumorkontrolle [42]. Die Resektion des HCC vor der Lebertransplantation dient somit nicht nur zur Kontrolle des Tumorwachstums sowie der Gefäßinvasion während der Wartezeit und damit dem Verbleib auf der Warteliste, sondern auch der tumorbiologischen Selektion der Patienten.

Cherqui et al. [43] untersuchten 67 Patienten mit HCC in Zirrhose innerhalb der Mailand-Kriterien, die primär leberreseziert wurden, anstatt für eine Transplantation gelistet zu werden. Die postoperative Morbidität betrug 34\% und die Mortalität 4,5\%. Nach einem mittleren Follow-up von 4,8 Jahren hatten $54 \%$ der Patienten ein intrahepatisches Rezidiv. Von diesen Patienten wurden wiederum 44\% transplantiert, wenn sie die Altersgrenze nicht überschritten und das Rezidiv innerhalb der Mailand-Kriterien war. Das Gesamtund das rezidivfreie Überleben in diesem Patientenkollektiv betrug 72 und $44 \%$. Somit konnte trotz der signifikanten Rezidivrate mit einem engmaschigen postoperativen Monitoring eine sogenannte «Rescue»-Transplantation bei $61 \%$ der Pati- enten immer noch durchgeführt werden. Dadurch stehen mehr Organe für andere Patienten zur Lebertransplantation zur Verfügung. Um dieses pragmatische und organsparende Vorgehen $\mathrm{zu}$ belegen, muss allerdings eine randomisierte kontrollierte Studie durchgeführt werden.

\section{Leberresektion als definitive Therapie}

Eine Transplantation kommt jedoch für ältere Patienten (>75 Jahre) auch als «Rescue»-Therapie nicht infrage. Und auch jüngere Patienten mit HCC außerhalb der Mailand-Kriterien, die somit nicht unter eine Sonderregelung fallen, warten zu lange auf eine Transplantation. Somit muss die Leberresektion als definitive Therapie zunehmend in Betracht gezogen werden. Dies gilt auch für Patienten mit HCC innerhalb der Mailand-Kriterien, die aber auch aufgrund des Organmangels so lange warten, dass das Risiko eines Tumorprogresses während der Wartezeit weiter zunimmt. Resektionen werden deshalb auch zunehmend bei fortgeschrittenen Tumoren (BCLC-Stadium B und C) durchgeführt $[44,45]$. Torzilli et al. [44] konnten zeigen, dass bei sorgfältiger Patientenselektion und adäquater parenchymsparender chirurgischer Technik unter Anwendung des IOUS große Tumoren mit vaskulärer Infiltration sowie niedriger Morbidität und Mortalität reseziert werden können [44]. Das Gesamtüberleben der 28 Patienten im BCLC-Stadium C nach der Resektion betrug nach 3 Jahren $70 \%$, das rezidivfreie Überleben allerdings nur 34\%. Auch andere aktuelle Beobachtungsstudien konnten zeigen, dass die über die letzten Dekaden deutlich besser werdenden Langzeitergebnisse der Leberresektion inzwischen bezüglich des Gesamtüberlebens mit der Lebertransplantation vergleichbar sind [45-48]. Dies wurde sowohl für Patienten mit HCC innerhalb der Mailand-Kriterien [47] als auch für HCC außerhalb der Mailand-Kriterien [45] gezeigt, gilt allerdings nur für das Gesamtüberleben und nicht für das rezidivfreie Überleben [35]. Zur Verbesserung des Gesamtüberlebens benötigen die Patienten eine lebenslange engmaschige postoperative Nachsorge mit optimierter Schnittbildgebung und sofortiger Therapie des Rezidivs [46]. Effektivere lokal ablative Verfahren als Alternative zur Re-Resektion sind hierfür notwendig.

Zusammenfassend lässt sich sagen, dass eine Erweiterung bzw. Revision der BCLC-Kriterien hin zur Resektion auch in fortgeschritteneren Stadien für einige Patientengruppen sinnvoll erscheint. Hierfür ist die Durchführung einer prospektiven Studie notwendig.

\section{Disclosure Statement}

HJS hat Vortragshonorare und Reisekostenerstattung von Novartis, Astellas, Pfizer und Roche erhalten. Er erfährt Forschungsunterstützung durch Pfizer und Novartis.

SAF erhielt Vortragshonorare und Reisenkostenerstattung bzw. bekommt Forschungsunterstützung von Novartis, Astellas, Roche und Pfizer. 


\section{Literatur}

1 Bruix J, Llovet JM: Prognostic prediction and treatment strategy in hepatocellular carcinoma. Hepatology 2002;35:519-524.

2 Eguchi S, Kanematsu T, Arii S, Omata M, Kudo M, Sakamoto M, Takayasu K, Makuuchi M, Matsuyama Y, Monden M: Recurrence-free survival more than 10 years after liver resection for hepatocellular carcinoma. Br J Surg 2011;98:552-557.

3 Makuuchi M, Kokudo N, Arii S, Futagawa S, Kaneko S, Kawasaki S, Matsuyama Y, Okazaki M, Okita K, Omata M, Saida Y, Takayama T, Yamaoka Y: Development of evidence-based clinical guidelines for the diagnosis and treatment of hepatocellular carcinoma in Japan. Hepatol Res 2008;38:37-51.

4 Benson AB III, Abrams TA, Ben-Josef E, et al: NCCN clinical practice guidelines in oncology: hepatobiliary cancers. J Natl Compr Canc Netw 2009;7:350-391.

5 Poon D, Anderson BO, Chen LT, Tanaka K, Lau WY, Van CE, Singh H, Chow WC, Ooi LL, Chow P, Khin MW, Koo WH: Management of hepatocellular carcinoma in Asia: consensus statement from the Asian Oncology Summit 2009. Lancet Oncol 2009;10:1111-1118.

6 Lang H, Sotiropoulos GC, Brokalaki EI, Schmitz KJ, Bertona C, Meyer G, Frilling A, Paul A, Malago M, Broelsch CE: Survival and recurrence rates after resection for hepatocellular carcinoma in noncirrhotic livers. J Am Coll Surg 2007;205: 27-36.

7 Laurent C, Blanc JF, Nobili S, Sa CA, le Bail B, Bioulac-Sage P, Balabaud C, Capdepont M, Saric $\mathrm{J}$ : Prognostic factors and longterm survival after hepatic resection for hepatocellular carcinoma originating from noncirrhotic liver. J Am Coll Surg 2005;201:656-662.

8 Dupont-Bierre E, Compagnon P, Raoul JL, Fayet G, de Lajarte-Thirouard AS, Boudjema K: Resection of hepatocellular carcinoma in noncirrhotic liver: analysis of risk factors for survival. J Am Coll Surg 2005;201:663-670.

9 Stipa F, Yoon SS, Liau KH, Fong Y, Jarnagin WR, D'Angelica M, Abou-Alfa G, Blumgart LH, DeMatteo RP: Outcome of patients with fibrolamellar hepatocellular carcinoma. Cancer 2006;106:1331-1338.

10 Schlitt HJ, Neipp M, Weimann A, Oldhafer KJ, Schmoll E, Boeker K, Nashan B, Kubicka S, Maschek H, Tusch G, Raab R, Ringe B, Manns MP, Pichlmayr R: Recurrence patterns of hepatocellular and fibrolamellar carcinoma after liver transplantation. J Clin Oncol 1999;17:324-331.

11 McWilliams JP, Yamamoto S, Raman SS, Loh CT, Lee EW, Liu DM, Kee ST: Percutaneous ablation of hepatocellular carcinoma: current status. J Vasc Interv Radiol 2010;21(suppl 8):S204-S213.

12 Sorensen M, Frisch K, Bender D, Keiding S: The potential use of 2-[ $\left.{ }^{18} \mathrm{~F}\right]$ fluoro-2-deoxy-D-galactose as a PET/CT tracer for detection of hepatocellular carcinoma. Eur J Nucl Med Mol Imaging 2011;38: 1723-1731.

13 Seo S, Hatano E, Higashi T, Hara T, Tada M, Tamaki N, Iwaisako K, Ikai I, Uemoto S: Fluorine-18 fluorodeoxyglucose positron emission tomography predicts tumor differentiation, P-glycoprotein expression, and outcome after resection in hepatocellular carcinoma. Clin Cancer Res 2007;13(Pt 1): 427-433.
14 Loss M, Schneider J, Uller W, Wiggermann P, Scherer MN, Jung W, Schlitt HJ, Stroszczynski C, Jung EM: Intraoperative high resolution linear contrast enhanced ultrasound (IOUS) for detection of microvascularization of malignant liver lesions before surgery or radiofrequeny ablation. Clin Hemorheol Microcirc 2012;50:65-77.

15 Loss M, Jung EM, Scherer MN, Farkas SA, Schlitt HJ: Chirurgische Therapie von Lebermetastasen. Chirurg 2010;81:533-541.

16 Poon RT, Fan ST, Ng IO, Wong J: Significance of resection margin in hepatectomy for hepatocellular carcinoma: a critical reappraisal. Ann Surg 2000; 231:544-551.

17 Shi M, Guo RP, Lin XJ, Zhang YQ, Chen MS, Zhang CQ, Lau WY, Li JQ: Partial hepatectomy with wide versus narrow resection margin for solitary hepatocellular carcinoma: a prospective randomized trial. Ann Surg 2007;245:36-43.

18 Minagawa M, Makuuchi M, Takayama T, Kokudo $\mathrm{N}$ : Selection criteria for repeat hepatectomy in patients with recurrent hepatocellular carcinoma. Ann Surg 2003;238:703-710.

19 Fong Y, Sun RL, Jarnagin W, Blumgart LH: An analysis of 412 cases of hepatocellular carcinoma at a Western center. Ann Surg 1999;229:790-799.

20 Gouillat C, Manganas D, Saguier G, Duque-Campos R, Berard P: Resection of hepatocellular carcinoma in cirrhotic patients: longterm results of a prospective study. J Am Coll Surg 1999;189:282-290.

21 Lang H, Broelsch CE: Resektion und Transplantation bei Lebertumoren. Internist (Berl) 2007;48: 30-39.

22 Kim BW, Kim YB, Wang HJ, Kim MW: Risk factors for immediate post-operative fatal recurrence after curative resection of hepatocellular carcinoma. World J Gastroenterol 2006;12:99-104.

23 Shah SA, Greig PD, Gallinger S, Cattral MS, Dixon E, Kim RD, Taylor BR, Grant DR, Vollmer CM: Factors associated with early recurrence after resection for hepatocellular carcinoma and outcomes. J Am Coll Surg 2006;202:275-283.

24 Liu H, Zhu S: Present status and future perspectives of preoperative portal vein embolization. Am J Surg 2009;197:686-690.

25 Abulkhir A, Limongelli P, Healey AJ, Damrah O, Tait P, Jackson J, Habib N, Jiao LR: Preoperative portal vein embolization for major liver resection: a meta-analysis. Ann Surg 2008;247:49-57.

26 Schnitzbauer AA, Lang SA, Goessmann H, Nadalin S, Baumgart J, Farkas SA, Fichtner-Feigl S, Lorf T, Goralcyk A, Horbelt R, Kroemer A, Loss M, Rummele P, Scherer MN, Padberg W, Konigsrainer A, Lang H, Obed A, Schlitt HJ: Right portal vein ligation combined with in situ splitting induces rapid left lateral liver lobe hypertrophy enabling 2-staged extended right hepatic resection in smallfor-size settings. Ann Surg 2012;255:405-414.

27 Aloia TA, Vauthey JN: Associating liver partition and portal vein ligation for staged hepatectomy (ALPPS): what is gained and what is lost? Ann Surg 2012;256:e9.

28 Sala S, Ardiles V, Ulla M, Alvarez F, Pekolj J, de Santibañes E: Our initial experience with ALPPS technique: encouraging results. Updates Surg 2012; 64:167-172.

29 Conrad C, Shivathirthan N, Camerlo A, Strauss C, Gayet B: Laparoscopic portal vein ligation with in situ liver split for failed portal vein embolization. Ann Surg 2012;256:e14-e15.
30 Lin NC, Nitta H, Wakabayashi G: Laparoscopic major hepatectomy: a systematic literature review and comparison of 3 techniques. Ann Surg 2013; 257:205-213.

31 Dagher I, Belli G, Fantini C, Laurent A, Tayar C, Lainas P, Tranchart H, Franco D, Cherqui D: Laparoscopic hepatectomy for hepatocellular carcinoma: a European experience. J Am Coll Surg 2010;211:16-23.

32 Cheung TT, Poon RT, Yuen WK, Chok KS, Jenkins CR, Chan SC, Fan ST, Lo CM: Long-term survival analysis of pure laparoscopic versus open hepatectomy for hepatocellular carcinoma in patients with cirrhosis: a single-center experience. Ann Surg 2013;257:506-511.

33 Mazzaferro V, Regalia E, Doci R, Andreola S, Pulvirenti A, Bozzetti F, Montalto F, Ammatuna M, Morabito A, Gennari L: Liver transplantation for the treatment of small hepatocellular carcinomas in patients with cirrhosis. N Engl J Med 1996;334: 693-699.

34 Mazzaferro V, Chun YS, Poon RT, Schwartz ME, Yao FY, Marsh JW, Bhoori S, Lee SG: Liver transplantation for hepatocellular carcinoma. Ann Surg Oncol 2008;15:1001-1007.

35 Mazzaferro V, Llovet JM, Miceli R, et al: Predicting survival after liver transplantation in patients with hepatocellular carcinoma beyond the Milan criteria: a retrospective, exploratory analysis. Lancet Oncol 2009;10:35-43.

36 Jonas S, Bechstein WO, Steinmuller T, Herrmann M, Radke C, Berg T, Settmacher U, Neuhaus P: Vascular invasion and histopathologic grading determine outcome after liver transplantation for hepatocellular carcinoma in cirrhosis. Hepatology 2001;33:1080-1086.

37 Yi NJ, Suh KS, Kim T, Kim J, Shin WY, Lee KU: Current role of surgery in treatment of early stage hepatocellular carcinoma: resection versus liver transplantation. Oncology 2008;75(suppl 1):124-128.

38 Yao FY, Xiao L, Bass NM, Kerlan R, Ascher NL, Roberts JP: Liver transplantation for hepatocellular carcinoma: validation of the UCSF-expanded criteria based on preoperative imaging. Am J Transplant 2007;7:2587-2596.

39 Clavien PA, Lesurtel M, Bossuyt PM, Gores GJ, Langer B, Perrier A: Recommendations for liver transplantation for hepatocellular carcinoma: an international consensus conference report. Lancet Oncol 2012;13:e11-e22.

40 Schlitt HJ, Schnitzbauer AA: Hepatocellular carcinoma: agents and concepts for preventing recurrence after curative treatment. Liver Transpl 2011;17(suppl 3):S10-S12.

41 Schnitzbauer AA, Schlitt HJ, Geissler EK: Influence of immunosuppressive drugs on the recurrence of hepatocellular carcinoma after liver transplantation: a gap between basic science and clinical evidence. Transplantation 2011;91:1173-1176.

42 Belghiti J, Cortes A, Abdalla EK, Regimbeau JM, Prakash K, Durand F, Sommacale D, Dondero F, Lesurtel M, Sauvanet A, Farges O, Kianmanesh R Resection prior to liver transplantation for hepatocellular carcinoma. Ann Surg 2003;238:885-892.

43 Cherqui D, Laurent A, Mocellin N, Tayar C, Luciani A, Van Nhieu JT, Decaens T, Hurtova M, Memeo R, Mallat A, Duvoux C: Liver resection for transplantable hepatocellular carcinoma: long-term survival and role of secondary liver transplantation. Ann Surg 2009;250:738-746. 
44 Torzilli G, Donadon M, Marconi M, Palmisano A, Del Fabbro D, Spinelli A, Botea F, Montorsi M: Hepatectomy for stage $\mathrm{B}$ and $\mathrm{C}$ hepatocellular carcinoma in the Barcelona Clinic Liver Cancer classification: results of a prospective analysis. Arch Surg 2008;143:1082-1090.

45 Canter RJ, Patel SA, Kennedy T, D'Angelica MI, Jarnagin WR, Fong Y, Blumgart LH, Freeman RB DeMatteo RP, Abt PL: Comparative analysis of outcome in patients with hepatocellular carcinoma exceeding the Milan criteria treated with liver transplantation versus partial hepatectomy. Am J Clin Oncol 2011;34:466-471.
46 Fan ST, Mau Lo C, Poon RT, Yeung C, Leung Liu C, Yuen WK, Ming Lam C, Ng KK, Ching Chan S: Continuous improvement of survival outcomes of resection of hepatocellular carcinoma: a 20-year experience. Ann Surg 2011;253:745-758.

47 Chua TC, Saxena A, Chu F, Morris DL: Hepatic resection for transplantable hepatocellular carcinoma for patients within Milan and UCSF criteria. Am J Clin Oncol 2012;35:141-145.
48 Facciuto ME, Rochon C, Pandey M, RodriguezDavalos M, Samaniego S, Wolf DC, Kim-Schluger L, Rozenblit G, Sheiner PA: Surgical dilemma: liver resection or liver transplantation for hepatocellular carcinoma and cirrhosis. Intention-to-treat analysis in patients within and outwith Milan criteria. HPB (Oxford) 2009;11:398-404. 